\title{
Apoyo educativo y patrón de vida en el paciente con tratamiento de hemodiálisis
}

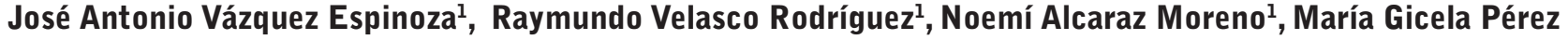 \\ Hernández ${ }^{1}$, Leticia Casique Casique ${ }^{2}$ \\ ${ }^{1}$ Facultad de Enfermería, Universidad de Colima. México. ${ }^{2}$ Facultad de Enfermería, Universidad de Guanajuato. \\ México
}

\section{Resumen}

Introducción: Actualmente el número creciente de personas en tratamiento de hemodiálisis aunado al incremento de incidencia de complicaciones agudas, pone de manifiesto implementar estrategias que favorezcan conductas o hábitos saludables disminuyendo las consecuencias personales, familiares y gastos institucionales.

Objetivos: Determinar la influencia del apoyo educativo de enfermería en el patrón de vida del paciente con insuficiencia renal crónica en tratamiento de hemodiálisis.

Metodología: Estudio pre-experimental donde participaron 22 pacientes en hemodiálisis, se diseñó y ejecutó un programa educativo bajo el referente teórico de Orem con enfoque en tres dimensiones: Responsabilidad en Salud, Alimentación e Higiene, consistió en diez sesiones de media hora dos veces por semana durante la primera hora de tratamiento del paciente.

Resultados: Edad promedio de $36.4 \pm 16.7$ años, con igual distribución tanto para hombres como mujeres $(n=11)$; el $68.1 \%(n=15)$ de los pacientes son económicamente activos, reportando un tiempo promedio de $20.6 \pm 15.6$ meses de tratamiento en hemodiálisis.
Se obtuvo un valor de mediana en la fase de pre-intervención de 92 y, de 107.5 en la fase post-intervención. Con una diferencia estadísticamente significativa entre ambas de $p<0.001$.

Conclusión: El 100\% de los sujetos mantiene un patrón de vida protector, lo que sugiere que el Apoyo Educativo de Enfermería aumenta el patrón de vida del paciente con tratamiento de hemodiálisis. Lo que hace trascendente el papel de enfermería en la promoción de conductas generadoras de salud.

PALABRAS CLAVE

- ENFERMERÍA

- APOYO EDUCATIVO

- HEMODIÁLISIS

Educational support and pattern-of-life in the patient treated with hemodialysis

\begin{abstract}
Introduction: The increase in the number of persons in hemodialysis treatment, together with the greater incidence of acute complications, illustrate the necessity of designing strategies that emphasize the promotion of health-generating behavior in these patients, reducing the consequences personal, family and institutional costs.

Aims: To determine the influence of educational support provided by the nursing professional on the pattern-of-life of the patient with hemodialysis treatment.
\end{abstract}


Methodology: A pre-experimental study was conducted on 22 patients undergoing hemodialysis treatment. An educational program based on the Orem theoretical reference was designed and put into practice, concentrating on three aspects: responsibility in relation to health, hygiene, and diet. The program consisted of a total of ten half-hour sessions, each one given twice a week for a period of five weeks during the patient's first hour of hemodialysis treatment.

Results: The mean age of the patients was $36.4 \pm$ 16.7 years. A total of $68.1 \%(n=15)$ of the patients were economically active, and the mean time of the patient in the hemodialysis treatment program was $20.6 \pm 15.6$ months. The median questionnaire score was 92 in the pre-intervention phase and 107.5 in the post-intervention phase. With a statistically significant difference of $p<0.001$.

Conclusion: One hundred percent of the subjects maintained a protective pattern-of-life, suggesting that educational support provided by the nursing professional increased the pattern-of-life score of the patients treated with hemodialysis.

\section{KEYWORDS}

- EDUCATIONAL SUPPORT

- HEMODIALYSIS

- NURSING

\section{Introducción}

El autocuidado es una función reguladora que las personas deben, deliberadamente, llevar a cabo por si solas para mantener su vida, salud, desarrollo y bienestar ${ }^{1}$, lo que implica que el autocuidado se debe aprender y desarrollar de manera continua, y conforme a los requisitos reguladores de cada persona.

Orem, señala que en todo acto de cuidado es importante considerar los factores condicionantes básicos, ya que son los que caracterizan a las personas como elementos que hablan sobre sus cualidades y rasgos que influyen en la individualidad de las personas ${ }^{2}$. El entendimiento de éstos, permitirán a la enfermera mejorar su ayuda y colaborar en la realización de autocuidado terapéutico, ayudando a predecir o explicar variaciones en los requisitos de autocuidado o en las capacidades de las personas para realizar el cuidado.
Lo anterior es trascendente, porque el profesional de enfermería que realiza cuidado a pacientes en tratamiento de hemodiálisis (HD), debe tener presente en todo momento que es imprescindible estar valorando al paciente porque el procedimiento al que se somete puede tener como consecuencia complicaciones súbitas tan graves como la muerte, y debe tener la capacidad de identificar las necesidades de autocuidado en función de las desviaciones que experimente su estado de salud.

Por ello, el apoyo al patrón de vida, definido como las acciones normales de autocuidado que una persona realiza ${ }^{3}$, puede verse afectado porque en el paciente en $H D$, su nivel de energía se ve comprometido por efectos propios de la enfermedad, tales como anemia y elevación de los compuestos nitrogenados.

Tan solo por ejemplificar, cuando el patrón de vida de una persona implique una adecuación de la ingesta de líquidos por estar en tratamiento de HD, el mantenimiento de un equilibrio hídrico se vuelve una necesidad prioritaria, y la forma de satisfacerlo es una ingesta de líquidos en función del volumen de eliminación urinaria diario, aun cuando se apoye con todas las medidas y estrategias necesarias para lograr un consumo restringido de líquidos, el paciente, puede no ser capaz de admitir las medidas necesarias en su demanda de autocuidado terapéutico, influenciado por el nivel educativo, la ocupación, el acceso a los recursos para seguir las indicaciones, la religión y costumbres sociales.

Por otro lado, la Insuficiencia Renal Crónica (IRC) constituye un problema de salud creciente a nivel mundial. La diabetes mellitus y la hipertensión arterial se encuentran entre las principales etiologías. La Organización Mundial de la Salud estima que hay aproximadamente $\mathbf{1 . 0 0 0}$ millones de personas en todo el mundo con hipertensión arterial y se espera que la cifra llegue a 1.500 millones para el 2025, prevaleciendo un incremento del $80 \%$ de los casos en los países de América Latina y por consecuencia mayor número de personas con IRC4.

La situación antes mencionada pone de manifiesto la necesidad de implementar estrategias que favorezcan conductas o hábitos saludables en el paciente con tratamiento de HD con el objeto de disminuir las consecuencias personales, familiares y gastos institucionales debido a las complicaciones derivadas del proceso de la enfermedad renal. Existen varias limitaciones ante una enfermedad crónica, y una de ellas es la deficiente educación para promover el autocuidado, la escasa 
promoción de un estilo de vida saludable y la inadecuada comunicación entre los pacientes y los equipos de salud que lo atienden ${ }^{5}$.

Se sabe que el paciente que recibe tratamiento de HD, tendrá ciertas restricciones en la ingestión de líquidos, proteínas y alimentos ricos en potasio, fósforo y sodio ${ }^{6}$, por lo tanto el acompañamiento en el manejo de la dieta se puede considerar como un factor protector frente a la pérdida de sustancias nutritivas. Sin embargo, es precisamente la falta de adherencia al tratamiento lo que se estima en un $45 \%$ sobre todo cuando existen cambios radicales en los hábitos o estilos de vida ${ }^{7}$.

Con base a lo anterior, es fundamental que el profesional de enfermería conozca, intervenga y asuma un papel determinante en la educación sanitaria del paciente y más aún, con el riesgo elevado que tiene de padecer deterioros alimenticios, esto con el propósito de que el paciente sea responsable de su propio patrón de vida protector.

\section{Objetivo}

El objetivo fue determinar la influencia del apoyo educativo de enfermería en el patrón de vida del paciente con insuficiencia renal crónica en tratamiento de HD.

\section{Material y Método}

Estudio pre-experimental con diseño antes-después cuya población de estudio estuvo conformada por 22 pacientes que reunieron los criterios de selección y que acudían a tratamiento al Centro Estatal de Hemodiálisis de la ciudad de Colima, en México. La selección de los sujetos de estudio se realizó mediante un muestreo aleatorio simple, seleccionando sujetos de ambos géneros que estuviesen hemodinámicamente estables, sin terapia nutricional ni episodios de infección vigentes.

\section{Instrumento}

Derivado de una búsqueda sistemática de la información, no se encontró instrumento alguno que abordara las tres dimensiones del patrón de vida, por lo que, se elaboró un instrumento al cual se le denominó "Cuestionario sobre el patrón del vida del paciente con tratamiento de hemodiálisis" (anexo 1) el cual, fue piloteado con 10 pacientes con características similares a nuestros sujetos de estudio. Se midió confiabilidad del mismo obteniendo un coeficiente alfa-Cronbach de 0.880 .
La estructura del instrumento contó con dos apartados: el primero con datos personales que indagaron información respecto a edad, género, ocupación, religión, escolaridad, edad de diagnóstico de la IRC y antigüedad en tratamiento de hemodiálisis; el segundo apartado, se denomina Patrón de Vida, y estuvo compuesto por 24 ítems que valoran las tres dimensiones del patrón de vida: responsabilidad en salud, higiene y alimentación.

Interpretación: La escala de medición es de tipo Likert con cinco opciones de respuesta y una puntuación que va de un máximo de 120 a un mínimo de 24 puntos. Se determinó un Patrón de vida "en riesgo" con puntuaciones de 24 a 71 puntos y Patrón de vida "protector" con 72 a 120 puntos.

Un patrón de vida en riesgo sugiere que la persona no cuenta con los conocimientos adecuados para favorecer conductas generadoras de salud.

Un patrón de vida protector sugiere que la persona cuenta con los conocimientos adecuados para favorecer conductas generadoras de salud.

\section{Aplicación de la intervención}

Previa autorización del comité de ética e investigación del Centro Estatal de Hemodiálisis que en la ciudad de Colima recibe el nombre de comité de calidad y seguridad del paciente, así como la confirmación de participación de cada uno de los pacientes, a los que se les proporcionó carta de consentimiento informado, mismas que signaron en presencia de un familiar, elaborada con base a lineamientos internacionales ${ }^{8}$ y nacionales como la Ley General de Salud de México ${ }^{9}$, se procedió a la aplicación de la fase de pre-intervención que consistió en contestar el cuestionario sobre el Patrón de vida en los primeros treinta minutos de iniciado el tratamiento de hemodiálisis. Posteriormente se implementó el apoyo educativo (fase de intervención) que consistió en proporcionar 10 sesiones individualizadas, en la primera hora de tratamiento de hemodiálisis, debido a que en ese tiempo se ha observado la menor incidencia de complicaciones agudas. Cada sesión tuvo una duración de treinta minutos, dos veces por semana durante cinco semanas. Este apoyo educativo se proporcionó en cada uno de los 10 módulos de hemodiálisis general cuya área de superficie por paciente era de 5.5 metros cuadrados los cuales, estaban delimitados por una pared favoreciendo con ello un cuidado individualizado con distracciones mínimas.

Las sesiones de apoyo educativo se proporcionaron fomentando la relación paciente - enfermero a través de 
una conversación donde mediante preguntas dirigidas, se activaron los conocimientos previos de los pacientes para identificar sus necesidades en función de las tres dimensiones del patrón de vida (responsabilidad en salud, higiene y alimentación). Se les mencionó sobre la importancia del aprovechamiento que deben tener del equipo multidisciplinario que se tiene en el centro de hemodiálisis, la forma en cómo cuidar en sus casas el acceso vascular, la identificación de signos de alarma en su cuerpo; por otro lado, la trascendencia de la higiene para la disminución de riesgos de infecciones; por último, el adecuado control de líquidos que deben tener. Se indagó sobre la disposición del tipo de alimentos en sus lugares de origen, para hacerles saber la forma en cómo pueden consumirlos bajo ciertas formas de preparación.

Finalmente, en la sesión de hemodiálisis número once se procedió a aplicar la fase de post-intervención que consistió en contestar nuevamente el "cuestionario sobre el patrón de vida" previamente aplicado en la fase de pre-intervención.

\section{Análisis estadístico}

El análisis de la información demográfica con base en el instrumento utilizado, fue realizado con estadística descriptiva (frecuencias, porcentajes, promedios y desviación estándar). Para el contraste de hipótesis se utilizó prueba de Wilcoxon mediante la comparación de medianas de la fase pre-intervención con la fase post-intervención. Las diferencias entre ambas fases fue considerada estadísticamente significativa cuando $p \leq 0.05$.

\section{Resultados}

\section{Datos demográficos:}

La distribución por género fue igual para masculinos como femeninos $(n=11)$; el $68.1 \% \quad(n=15)$ de los pacientes se encontró económicamente activo; se encontró que el $95.5 \%(n=21)$ de los pacientes profesan la religión católica; respecto a la educación básica o elemental, el 77.3 ( $n=17)$ tiene primaria o secundaria terminada; Edad promedio de $36.4 \pm 16.7$ años; la edad promedio de diagnóstico de la IRC fue a los 34.5 años \pm 17.7 ; se encontró un tiempo promedio de $20.6 \pm 15.6$ meses de tratamiento en el programa de hemodiálisis.
Patrón de vida

La Tabla 1 muestra el patrón de vida de las personas que participaron en el estudio, antes y después de la intervención denominada "apoyo educativo".

Tabla 1. Patrón de vida del paciente con hemodiálisis.

\begin{tabular}{l|l|ll|ll}
\hline Variable & Indicador & \multicolumn{3}{|l|}{ Fases } \\
& & \multicolumn{3}{|l|}{ Pre-intervención } & Post-intervención \\
PATRÓN & & & & & \\
DE VIDA & Riesgo & $\mathrm{f}$ & $\%$ & $\mathrm{f}$ & $\%$ \\
& & 1 & 4.5 & 0 & 0 \\
& Protector & 21 & 95.5 & 22 & 100 \\
\hline
\end{tabular}

Fuente: Cuestionario sobre Patrón de Vida del paciente con tratamiento de Hemodiálisis, $\mathrm{n}=22$.

En la Tabla 2, se muestra el comportamiento antes y después del apoyo educativo de cada una de las tres dimensiones en las que está estructurado el Patrón de Vida.

Tabla 2. Dimensiones del patrón de vida del paciente con hemodiálisis.

\begin{tabular}{|c|c|c|c|c|c|}
\hline \multirow[t]{2}{*}{ Dimensión } & \multirow[t]{2}{*}{ Indicador } & \multicolumn{2}{|c|}{ Pre-intervención } & \multicolumn{2}{|c|}{ Post-intervención } \\
\hline & & f & $\%$ & $\mathbf{f}$ & $\%$ \\
\hline \multirow{3}{*}{$\begin{array}{l}\text { RESPONSABILIDAD } \\
\text { EN LA SALUD }\end{array}$} & & & & & \\
\hline & Riesgo & 3 & 13.6 & 0 & 0 \\
\hline & Protector & 19 & 86.4 & 22 & 100 \\
\hline \multirow[t]{2}{*}{ HIGIENE } & Riesgo & 0 & 0 & 0 & 0 \\
\hline & Protector & 22 & 100 & 22 & 100 \\
\hline \multirow[t]{2}{*}{ ALIMENTACIÓN } & Riesgo & 3 & 13.6 & 0 & 0 \\
\hline & Protector & 19 & 84.4 & 22 & 100 \\
\hline
\end{tabular}

Fuente: Cuestionario sobre Patrón de Vida del paciente con tratamiento de Hemodiálisis, $n=22$.

Por otro lado, en la Tabla 3 se muestran los ítems en los que hubo un incremento de puntuación más evidente, se observa que hubo un mayor incremento en el ítem que corresponde al cuidado del acceso vascular. Además, se identificó que hubo un incremento en los ítems respecto a la dimensión de alimentación y responsabilidades propias de su enfermedad para incrementar el patrón de vida.

En la Tabla 4, se muestran las puntuaciones obtenidas en el Patrón de Vida antes y después de la intervención, observándose un incremento de 15 puntos en la mediana a favor del control post-intervención con una significancia estadística de $p<0.001$. 
Tabla 3. Ítems con mejor puntaje después del apoyo educativo.

\begin{tabular}{|l|l|c|c|c|}
\hline DIMENSIÓN & PTEMS & Pre prueba mediana & Post prueba mediana & Incremento \\
\hline Responsabilidad en salud & $\begin{array}{l}\text { *Conozco formas de proteger } \\
\text { mi acceso vascular para asearme } \\
\text { diario. } \\
\text { *Acudo a revisión médica solo } \\
\text { cuando me siento mal. }\end{array}$ & 63 & 39 \\
\hline Alimentación & $\begin{array}{l}\text { *Consumo en forma } \\
\text { limitada alimentos ricos } \\
\text { en carbohidratos (tortillas, } \\
\text { papa, arroz) en las comidas. } \\
\text { *Evito consumir alimentos ricos } \\
\text { en fósforo (frijoles, lentejas) } \\
\text { *Evito consumir lácteos } \\
\text { diariamente }\end{array}$ & 36 & 97 \\
26 & 71 & 99 \\
\hline
\end{tabular}

Fuente: Cuestionario sobre Patrón de Vida del paciente con tratamiento de Hemodiálisis, n=22.

Tabla 4. Influencia del apoyo educativo de enfermería en el patrón de vida.

\begin{tabular}{|l|l|l|l}
\hline PATRÓN DE VIDA & Mediana & Mínimo y Máximo & $\mathbf{P} *$ \\
\hline PRE & 92 & $\begin{array}{l}\text { Mínimo 70 } \\
\text { Máximo 114 }\end{array}$ & \\
\hline POST & 107.5 & $\begin{array}{l}\text { Mínimo 88 } \\
\text { Máximo 120 }\end{array}$ & $<0.001$ \\
\hline
\end{tabular}

* Calculada con Prueba de Wilcoxon.

\section{Discusión}

Actualmente la IRC condiciona que todo paciente ingresado en programa de terapia de HD se somete a "modificaciones de su vida social, restricción de líquidos, pérdida de esperanza de un trasplante renal, además de repercusiones emocionales y económicas ${ }^{\prime 10}$. Luego entonces después de perder el estado de salud y renunciar en la mayoría de las veces a proyectos de vida ya establecidos, puede resultar conveniente implementar programas educativos de enfermería en todo el proceso de salud - enfermedad, para propiciar cambios en función de las necesidades individuales de los pacientes.

En este estudio destaca el promedio de edad (36.4 años), considerado dentro de una etapa económicamente activa, dato que difiere con estudios europeos, donde se encontró que el promedio de edad de los pacientes que se encontraban en programa de HD, estaba por encima de 56.1 y 65 años respectivamente ${ }^{11-12}$, etapas consideradas post-productivas o de retiro laboral.
Por otro lado, el nivel de escolaridad que se reportó en el presente estudio, muestra que un $77.3 \%$ de la población tiene nivel elemental de estudios. Lo que resulta importante considerar a la hora de establecer estrategias educativas de cuidado porque un nivel de estudios mayor puede favorecer el incremento y optimización de los conocimientos que se proporcionan a través del apoyo educativo en los pacientes sometidas a HD, pero que por sí solo no es suficiente ${ }^{13}$, debiendo abarcar aspectos conductuales, cognoscitivos y afectivos como parte de las intervenciones educativas individualizadas. Sin embargo, el nivel de escolaridad alto se ha asociado con una mejor supervivencia de los pacientes en hemodiálisis ${ }^{14}$.

El tiempo de permanencia en tratamiento de HD, es un indicador importante de la supervivencia de los pacientes. En el presente estudio, la permanencia promedio fue de 20.6 meses lo que contrasta con otros estudios cuya permanencia en los programas de hemodiálisis está por encima de los 37 meses incrementando con ello la supervivencia de los pacientes ${ }^{15}$. Esta diferencia en los tiempos de permanencia radica en que muy probablemente en nuestro estudio, los pacientes deben contribuir a la compra del equipo básico (filtro, circuito extracorpóreo, concentrado ácido y bicarbonato) para la realización de su hemodiálisis, lo cual limitaría la asistencia y por ende la permanencia en el programa de HD.

Aunque los resultados del patrón de vida de los pacientes mejoraron con la intervención educativa, se 
constata que el proceso de HD y la misma enfermedad renal crónica, genera en los pacientes incomodidad, sufrimiento y déficit de conocimientos asociados a la enfermedad, interfiriendo con la adherencia al tratamiento y calidad de vida. Incluso se ha encontrado relación sobre la aparición de trastornos emocionales derivados de la incertidumbre respecto al tratamiento, limitaciones en la alimentación, cambios en las relaciones sociales y en el aspecto físico ${ }^{16}$.

Estas afecciones, disminuyen la colaboración de los pacientes respecto a su tratamiento y en algunos casos los conducen a adquirir conductas de manera negativa hacia los programas de hemodiálisis, de ahí que, Bonal Ruiz señale que la OMS reconoce que el autocuidado es la "piedra angular en la atención a pacientes con enfermedades crónicas 117 , sin embargo, se pueden agregar diversas causas que limitan las acciones de autocuidado entre las que se encuentran, la dificultad en el acceso a los servicios de salud, la mala coordinación de los mismos, y la deficiente educación del paciente por parte de los profesionales de la salud.

Por ello, pensamos que la mejora del autocuidado a través del fortalecimiento del patrón de vida del paciente en HD, podría favorecer la disminución de las complicaciones agudas, los internamientos no programados, costos para las instituciones, pero sobre todo, podría mejorar la calidad de vida de cada uno de los pacientes que ingresan en programa de sustitución renal ${ }^{18}$. Además creemos que mejoraría la incorporación a programas de inserción laboral o terapias ocupacionales, incrementando el afrontamiento y la adherencia al tratamiento.

Este estudio demuestra el efecto que tiene la intervención educativa en el patrón de vida del paciente, ya que las principales áreas en las que se influyó fueron las referentes al cuidado del acceso vascular, específicamente en las formas de protegerse el sitio de inserción de un catéter procurando mantener integro el apósito para la disminución de infecciones asociadas al acceso vascular; el favorecer la corresponsabilidad en salud que el paciente debe tener en su tratamiento, al identificar la necesidad de acudir a consultas no solo en situaciones de complicaciones, sino bajo un enfoque control o prevención para un mejor seguimiento de su tratamiento. $Y$ por último, la alimentación que resulta ser un elemento básico en el acompañamiento de todo paciente en HD, ha sido estudiada a través de intervenciones educativas que han favorecido el control bioquímico de valores plas- máticos de fósforo, potasio, colesterol y ácido úrico ${ }^{19}$, considerando siempre al cuidador principal. Además se ha demostrado con estudios de intervención el equilibrio hídrico y de presión arterial del paciente ${ }^{20}$.

Sin embargo, el reto más grande es mantener en el tiempo las intervenciones como parte de cada acto de cuidado que enfermería realiza. Teniendo en cuenta que el profesional de enfermería es el que mayor tiempo permanece en contacto directo con los pacientes durante sus sesiones de HD, pensamos que la enfermería tiene en cada una de estas sesiones una oportunidad valiosa para educar al paciente promoviendo conductas de autocuidado.

\section{Conclusión}

Como conclusión queda evidencia de la influencia positiva que el apoyo educativo tiene en el patrón de vida de los pacientes en HD para favorecer conductas de autocuidado.

Recibido: 18 octubre 16

Revisado: 03 noviembre 16

Modificado: 04 diciembre 16

Aceptado: 15 diciembre 16

\section{Bibliografía}

1. Marriner A, Raile M. Modelos y Teorías de Enfermería. Madrid, Elsevier-Mosby, $6^{a}$ edición; 2007.p. 269-270.

2. Orem DE. Modelo de Orem: Conceptos de Enfermería en la Práctica. Madrid, Masson; 1995.p..262-263.

3. Dennis CM. Self-Care Déficit Theory of Nursing: Concept San d Aplications. St. Louis, Mosby; 1997.p..3-5.

4. Organización Panamericana de la Salud. Base de datos de Mortalidad 2012. Health Intelligence Platform. (Acceso 23 octubre 2015). Disponible en: http://ais.paho.org/phip/viz/mort_ causasprincipales_It_oms.asp 
5. Sapag JC, Lange I, Campos S, Piette JD. Estrategias innovadoras para el cuidado y el autocuidado de personas con enfermedades crónicas en América Latina. Rev Panam de Salud Pública. 2010; 27(1):1-9. (Acceso 15 octubre 2015). Disponible en: http://www.scielosp.org/pdf/rpsp/v27n1/01.pdf

6. Pereira Feijoo MC, Queija Martínez L, Blanco Pérez A, Rivera Egusquiza IA, Martínez Maestro VE, Prada Monterrubio Z. Valoración del estado nutricional y consumo alimentario de los pacientes en terapia renal sustitutiva mediante hemodiálisis. Enferm Nefrol. 2015; 18(2): 103-111. (Acceso 5 noviembre 2015). Disponible en: http://scielo. isciii.es/pdf/enefro/v18n2/original4.pdf

7. Nabolsi MM, Wardam L, Al-Halabi J0. Quality of life, depression, adherence to treatment and illness perception of patients on haemodialysis. Int $\mathrm{J}$ Nurs Pract. 2015; 21(1): 1-10. (Acceso 28 julio de 2016). Disponible en: https://www.ncbi.nlm.nih. gov/pubmed/24124912

8. Asociación Médica Mundial. Declaración de Helsinki; Principios éticos para la investigación médica en seres humanos. Adoptado por la $64^{\mathrm{a}}$ Asamblea Médica Mundial. Fortaleza, Brasil. 2013. (Acceso 15 agosto de 2015). Disponible: http://www.isciii.es/ISCIII/es/contenidos/fdinvestigacion/fd-evaluacion/fd-evaluacion-eticainvestigacion/Declaracion-Helsinki-2013-Esp.pdf

9. Secretaria de Salud, Ley General de Salud. Título Quinto. México. (Acceso 15 agosto de 2015). Disponible en: http://www.salud.gob.mx/unidades/ cdi/legis/lgs/index-indice.htm

10. Rodríguez Ángel E, Campillo M, Avilés Esquivel M. Calidad de vida en pacientes con insuficiencia renal crónica y su familia. Rev Proc Psicológicos y Sociales. Hacer Psicología. 2013; 2(1-2). (Acceso 15 agosto de 2015). Disponible en: http://www. uv.mx/psicologia/files/2013/06/Calidad-de-vida.pdf

11. Galindo P, Pérez de la Cruz A, Cerezo S, Martínez $T$, López $P$, Asensio C. Malnutrición y mortalidad en pacientes en hemodiálisis. Nutr Hosp. 2001; 16 (1): 27-30. (Acceso 15 agosto de 2015). Disponible en: http://www.nutricionhospitalaria.com/pdf/3216. pdf

12. Arenas MD, Álvarez-Ude F, Angoso M, Berdud A, AntonlinA.etal.Valoración del grado de dependencia funcional de los pacientes en hemodiálisis (HD): estudio multicéntrico. Nefrología. 2006; 26 (5): 600-608. (Acceso 25 octubre de 2015). Disponible en: http://www.revistanefrologia.com/ es-publicacion-nefrologia-articulo-valoraciondel-grado-dependencia-funcional-los-pacienteshemodialisis-hd-estudio-X0211699506020351

13. Guerra Guerrero VT, Díaz Mujica $A E$, Vidal Albornoz K. La educación como estrategia para mejorar la adherencia de los pacientes en terapia dialítica. Rev Cubana Enferm. 2010; 26 (2): 52-62. (Acceso 2 noviembre 2015). Disponible en: $\quad$ http://scielo.sld.cu/scielo.php?script=sci_ arttext\&pid=S0864-03192010000200007

14. Khattak M, Sandhu G, Desilva R, GoldfarbRumyantzev A. Association of education level with dialysis outcome. Hemodialysis International 2012; 16:82-88. (Acceso 20 enero 2017). Disponible en: http://onlinelibrary.wiley.com/doi/10.1111/j.15424758.2011.00615.x/pdf

15. Valdés $C$, Miguel $M$, Rábano $M$, et al. Análisis del acuerdo entre la valoración que hacen los pacientes en hemodiálisis de su Calidad de Vida Relacionada con la Salud (CVRS) y la valoración que de ellos hace el personal de enfermería. Rev Soc Esp Enferm Nefrol. 2010; 13: 228234. (Acceso 2 noviembre 2015). Disponible en: $\quad$ http://scielo.isciii.es/scielo.php?script=sci_ arttext\&pid=S1139-13752010004400003

16. Sousa G, Dos Santos I, Bregman R. Clientes con enfermedad renal crónica: evaluación de enfermería sobre la competencia para el autocuidado. Esc Anna Nery 2007; 11 (1): 4451. (Acceso 3 marzo de 2016). Disponible en: http://www.scielo.br/scielo.php?pid=S1414$81452007000100006 \& \mathrm{script}=\mathrm{sci}$. abstract\&tlng=es

17. Bonal R, Cascaret X. ¿Autocuidado o autocontrol en enfermedades crónicas? Acercamiento a su análisis e interpretación. MEDISAN. 2009; 13 (1). (Acceso 3 marzo de 2016). Disponible en: http:// bvs.sld.cu/revistas/san/vol13_1_09/san18109.pdf

18. Torres Torrija CS. Diseño, implementación y evaluación de un programa de intervención educativa para pacientes con insuficiencia renal crónica. Rev Latín American Med Conductual. 2010; 1 (1): 3746. (Acceso 18 marzo 2016). Disponible en: http:// www.redalyc.org/pdf/2830/283021975005.pdf 
19. Torres Torradeflot MC, Gutiérrez Vilaplana JM, Craver L, Baigol Guilanya M. Resultado de la intervención Enseñanza: Dieta prescrita en la consulta de enfermedad renal crónica avanzada. Enferm Nefrol2016;19(1):12-19. (Acceso22enero 2017). Disponible en: http://www.revistaseden.org/ files/Articulos_3666_21riginal104822.pdf
20. Arribas Cobo P, Fernández Fuentes A, García Estévez $S$, Guimerá Ferré-Samá MA, Herrera Martín E, MartínezAranda MA. Los efectos de una intervención educativa sobre la ingesta de sal para disminuir la sobrehidratación y la hipertensión arterial en pacientes con hemodiálisis. Enferm Nefro 2013; 16 (supl 1). (Acceso 22 enero 2017). Disponible en: http://scielo.isciii.es/scielo.php?pid=S2254$28842013000500043 \&$ script=sci_arttext\&tlng=pt

Anexo 1. Cuestionario sobre Patrón de Vida del paciente con Hemodiálisis

Fecha:__ No. de cuestionario:

INTRODUCCIÓN: El objetivo es conocer las prácticas de comportamiento que las personas realizan para mantener su salud.

INSTRUCCIONES: Lea cuidadosamente las siguientes preguntas y coloque una $(X)$ en la opción de respuesta según consideres corresponda.

\section{I.- DATOS PERSONALES}

1.- Edad:

\begin{tabular}{|c|c|c|}
\hline 2.- Género & ( ) 1.Masculino & ( ) 2.Femenino \\
\hline \multirow[t]{4}{*}{ 3.- Ocupación } & ( ) l.Estudiante & ( ) 2.Ama de casa \\
\hline & ( ) 3.Empleado & ( ) 4.Comerciante \\
\hline & ( ) 5.Profesionista & ( ) 6.Campesino \\
\hline & ( ) 7.0tros: & \\
\hline \multirow[t]{3}{*}{ 4.- Estado civil } & ( ) 1.Soltero & ( ) 2.Casado \\
\hline & ( ) 3.Divorciado & ( ) 4.Separado \\
\hline & ( ) 5.Unión libre & ( ) 6.0tros: \\
\hline \multirow[t]{2}{*}{ 5.- Religión } & ( ) 1.Católico & ( ) 2.Cristiano \\
\hline & $\begin{array}{l}\text { ( ) } 3 . \text { Test. De Jehová } \\
\text { ( ) } 5 . \text { No creyente }\end{array}$ & ( ) 4.Protestante \\
\hline \multirow{3}{*}{ 6.- Escolaridad } & ( ) 1.Primaria & ( ) 2.Secundaria \\
\hline & ( ) 3. Bachillerato & ( ) 4.Profesional \\
\hline & ( ) 5.0tros: & \\
\hline \multirow[t]{3}{*}{ 7.- Nivel socioeconómico } & ( ) 1.Rico & ( ) 2.Media alta \\
\hline & ( ) 3.Media & ( ) 4.Media baja \\
\hline & ( ) 5.Pobre & ( ) 6.Pobreza extrema \\
\hline 8.- Edad de diagnóstico de la IRC & Años: & \\
\hline 9.- Tiempo con hemodiálisis & Años: & Meses: \\
\hline 10.- Aplicación de eritropoyetina & SI__ & NO \\
\hline
\end{tabular}

Instrucciones: Lea cuidadosamente cada enunciado, posteriormente seleccione solo una opción la cual será su respuesta, en seguida marque con una ( $\mathbf{X}$ ), considere que el cinco representa SIEMPRE, y el uno señala NUNCA. 


\section{[ José Antonio Vázquez Espinoza, et al ]}

Apoyo educativo y patrón de vida en el paciente con tratamiento de hemodiálisis

\section{PATRÓN DE VIDA}

\begin{tabular}{|c|c|c|c|c|c|c|}
\hline 11 & $\begin{array}{l}\text { Reactivo } \\
\text { Soy responsable de mi propia salud }\end{array}$ & $\begin{array}{c}\text { Nunca } \\
1\end{array}$ & $\begin{array}{l}\text { Casi Nunca } \\
\quad 2\end{array}$ & $\begin{array}{c}\text { A veces } \\
3\end{array}$ & $\begin{array}{c}\text { Casi siempre } \\
4\end{array}$ & $\begin{array}{l}\text { Siempre } \\
5\end{array}$ \\
\hline 12 & $\begin{array}{l}\text { Estoy al pendiente de lo que incremento } \\
\text { de peso entre una hemodiálisis y otra }\end{array}$ & & & & & \\
\hline 13 & $\begin{array}{l}\text { Conozco formas de protegerme } \\
\text { mi acceso para asearme diario }\end{array}$ & & & & & \\
\hline 14 & $\begin{array}{l}\text { Conozco como cuidar mi acceso } \\
\text { vascular para hemodiálisis }\end{array}$ & & & & & \\
\hline 15 & $\begin{array}{l}\text { Sigo el tratamiento } \\
\text { que me indica el médico }\end{array}$ & & & & & \\
\hline 16 & $\begin{array}{l}\text { Reviso mi piel en busca de sequedad, } \\
\text { herida o grieta }\end{array}$ & & & & & \\
\hline 17 & $\begin{array}{l}\text { Observo mi cuerpo constantemente } \\
\text { para identificar cambios anormales }\end{array}$ & & & & & \\
\hline 18 & $\begin{array}{l}\text { Acudo a revisión médica solo cuando } \\
\text { me siento mal }\end{array}$ & & & & & \\
\hline 19 & Me cambio de ropa diariamente & & & & & \\
\hline 20 & $\begin{array}{l}\text { Conozco el procedimiento adecuado } \\
\text { para asearme }\end{array}$ & & & & & \\
\hline 21 & $\begin{array}{l}\text { Cambio de sabanas, colchas } \\
\text { mi cama cada semana }\end{array}$ & & & & & \\
\hline 22 & $\begin{array}{l}\text { Evito tocar con las manos } \\
\text { mi acceso vascular }\end{array}$ & & & & & \\
\hline 23 & $\begin{array}{l}\text { Evito tener contacto constante } \\
\text { con animales domésticos }\end{array}$ & & & & & \\
\hline 24 & $\begin{array}{l}\text { Procuro mantener mi casa } \\
\text { en condiciones higiénicas para evitar } \\
\text { infectar mi acceso }\end{array}$ & & & & & \\
\hline 25 & $\begin{array}{l}\text { Evito exponer mi acceso al polvo, } \\
\text { humedad }\end{array}$ & & & & & \\
\hline 26 & $\begin{array}{l}\text { Mantengo bien cubierto mi acceso } \\
\text { para no exponerlo al ambiente }\end{array}$ & & & & & \\
\hline 27 & $\begin{array}{l}\text { Evito consumir alimentos que puedan } \\
\text { dañar mi salud }\end{array}$ & & & & & \\
\hline 28 & $\begin{array}{l}\text { Evito consumir alimentos ricos en potasio } \\
\text { (plátano, agua de coco) }\end{array}$ & & & & & \\
\hline 29 & $\begin{array}{l}\text { Evito consumir más de } 800 \mathrm{ml} \text { de líquido } \\
\text { por día }\end{array}$ & & & & & \\
\hline 30 & $\begin{array}{l}\text { Consumo alimentos que me ayudan } \\
\text { a no tener anemia (clara de huevo, carne) }\end{array}$ & & & & & \\
\hline 31 & $\begin{array}{l}\text { Evito consumir alimentos ricos en agua } \\
\text { que favorecen la acumulación de líquidos } \\
\text { en mi cuerpo }\end{array}$ & & & & & \\
\hline 32 & $\begin{array}{l}\text { Consumo en forma limitada } \\
\text { alimentos ricos en carbohidratos } \\
\text { (tortillas, papa, arroz) en las comidas }\end{array}$ & & & & & \\
\hline 33 & $\begin{array}{l}\text { Evito consumir alimentos ricos en fósforo } \\
\text { (frijoles, lentejas) }\end{array}$ & & & & & \\
\hline 34 & Evito consumir lácteos diariamente & & & & & \\
\hline
\end{tabular}

